\title{
I manuale scolastico e la trasposizione dei saperi storici. Un esempio di analisi
}

\author{
di Maila Pentucci
}

Apr 30, 2019 | Pensare la didattica $|\underline{0}|$

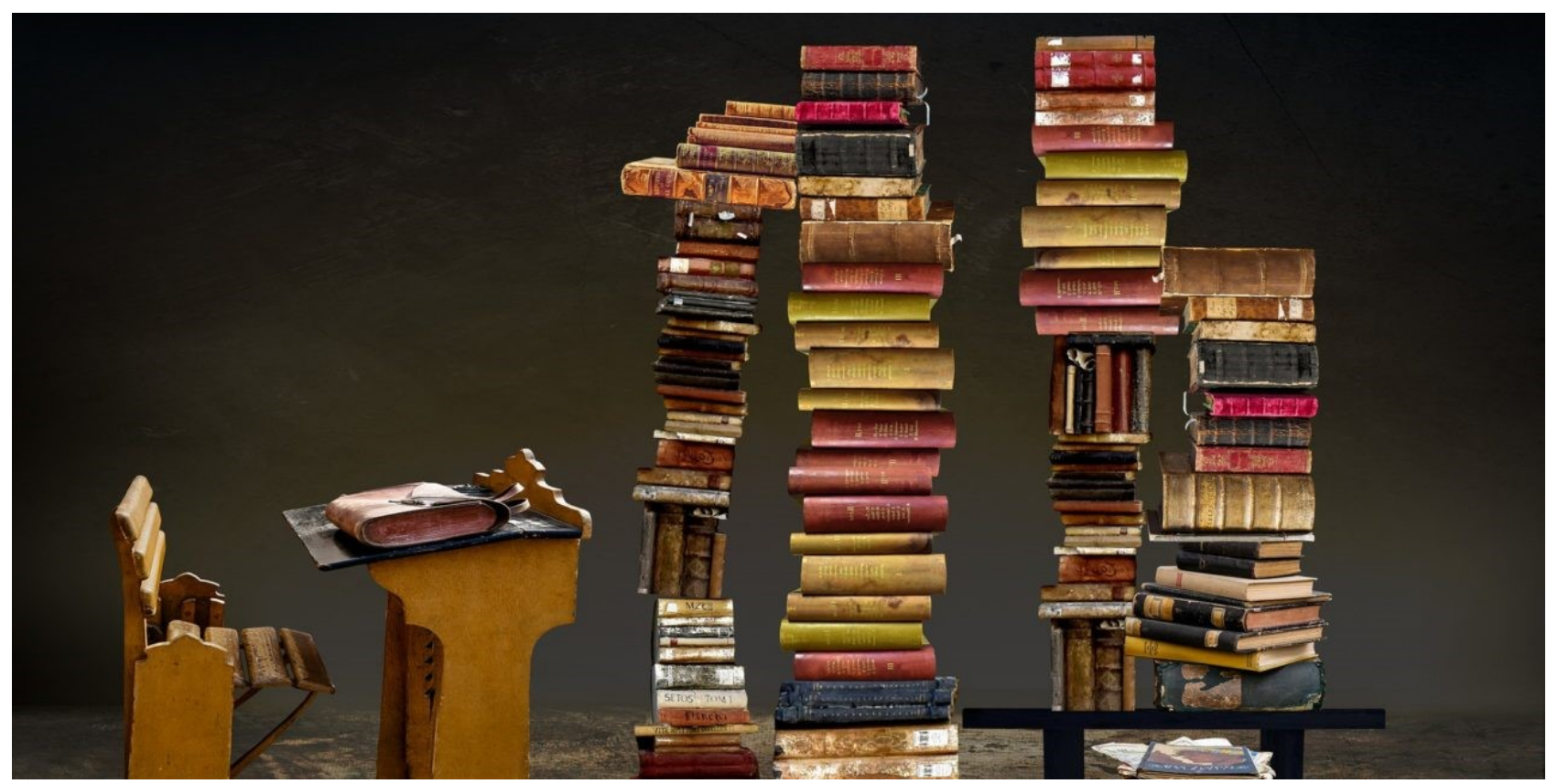

\section{Abstract}

Come viene realizzata la trasposizione didattica nei manuali di storia della scuola di base? In particolare, come vengono operazionalizzati i saperi e proposta la mediazione dei contenuti? Per rispondere a tali domande occorre prendere in carico il manuale non come oggetto per la ricerca, ma come oggetto di ricerca, centrale nei processi di insegnamento-apprendimento in quanto influenza la percezione del sapere storico degli insegnanti, mette in atto processi generativi per la progettazione e lo sviluppo di pratiche didattiche. Infatti, alcune ricorsività che caratterizzano l'insegnamento della storia sono ascrivibili alla strutturazione del sapere storico nei sussidiari e nei manuali di scuola secondaria: la concezione e l'operazionalizzazione del tempo, l'inclusione generalizzata di contenuti che diventano così, nella prassi, essenziali, la riproposizione di stereotipi culturali che la ricerca storica ha da lungo tempo smentito, lo spazio dato al racconto storico piuttosto che alla sua ricostruzione attraverso operazioni cognitive fondamentali, che tengano conto della grammatica e della sintassi proprie della disciplina esperta. A partire dall'analisi di alcuni elementi propri dei manuali si porta l'esempio di come essi comunichino l'idea di periodizzazione e operazionalizzino il tempo, dimensione fondamentale per l'insegnamento della storia, soprattutto nei primi anni.

\section{Introduzione}

Il manuale scolastico è una forma-libro complessa e sui generis: nel panorama editoriale si colloca entro un mercato atipico, che gioca secondo regole proprie e particolari e si rapporta con un 
pubblico di utenti fisso e non espandibile, entro il quale i decisori, ovvero coloro che orientano l'acquisto (i docenti), non corrispondono agli acquirenti ed agli utilizzatori finali (gli studenti).

Il rapporto tra insegnante e manuale è molto stretto e presenta un doppio feedback. La cultura di comunità ed i bisogni che essa manifesta influenzano profondamente la struttura, l'impostazione e la trattazione dei contenuti; nello stesso tempo la centralità del manuale nelle prassi della maggioranza dei docenti, sia per quanto riguarda la progettazione dei percorsi disciplinari, sia per quanto riguarda la mediazione didattica dei saperi in questione, contribuisce alla strutturazione della cultura scolastica, in veste sia di filtro che di generatore [Chervel 1998]: da un lato agisce da selettore nel processo di trasposizione didattica, dall'altro produce conoscenze negli alunni che lo utilizzano e negli insegnanti che lo considerano strumento di lavoro che può avviare ulteriori approfondimenti.

Il rapporto simbiotico tra docente e manuale è ben rappresentato da Lenoir [2006] che utilizza la metafora del martello: esistono varie tipologie di martello adeguate a operazioni differenti da compiere così come esistono manuali più o meno adatti per determinati bisogni educativi e cognitivi, ma la forza del martello sta principalmente nella mano di chi lo impugna. Per questo un ottimo manuale in mano a un pessimo insegnante può provocare danni, mentre un cattivo manuale utilizzato da un bravo insegnante può dare esiti molto positivi.

\section{Forme di trasposizione nei manuali}

Come entra il manuale nel processo di trasposizione didattica messo in atto dal docente? Ovvero, quali vincoli e inviti [Norman 1993] può porre ad un insegnante che lo ponga al centro del proprio processo di insegnamento-apprendimento, a livello di operazionalizzazione dei saperi storici?

Il buon manuale è quello che contiene saperi validati ed affidabili, non solo da mettere a disposizione per il processo di apprendimento dell'alunno, ma dei quali l'insegnante può approfittare nell'organizzare la propria progettazione. Di conseguenza il manuale deve presentare un certo livello di organizzazione didattica, pedagogica ed epistemologica, ma nello stesso tempo, deve lasciare al docente la libertà di effettuare la propria mediazione: «un'opera pedagogica che non sia realmente marcata pedagogicamente» [Vargas 2006, 273].

Il manuale, infatti, non dovrebbe imporre la propria linea né prevalere sull'intenzione del docente, né ostacolarlo o influenzarlo nel processo traspositivo, ma essere preso in considerazione solo a posteriori, per capire quali parti e quali modalità potrebbero, se ristrutturate, inserirsi a supporto di una trasposizione già realizzata dall'insegnante. Tuttavia, essendo uno strumento rigido e concluso, messo al centro del processo di insegnamento e apprendimento in quanto riconosciuto di una quasi esclusività nel mettere in discorso un tipo di sapere accessibile dal punto di vista scolastico, esso si inserisce nella catena traspositiva come referente primario per l'insegnante che progetta e pianifica e assume su di sé l'onere della mediazione.

Quali dimensioni o aspetti della trasposizione didattica vengono rappresentati nei manuali? Innanzi tutto la trasposizione epistemologica vera e propria: il manuale, nella sua natura di oggetto portatore e veicolatore di conoscenze, rappresenta la disciplina così come viene intesa nella scuola. Le istanze generaliste, per cui non può situarsi entro contesti di apprendimento specifici ma deve rispondere ad esigenze di mercato varie e vaste, fanno sì che le scelte epistemologiche siano convenzionali rispetto ad una tradizione non sempre in linea con i tempi, portatrici di eccessive banalizzazioni e 
semplificazioni, specchio, nel migliore dei casi, del paradigma scientifico e sociale dominante e più allineato con il senso comune che con gli orizzonti della ricerca.

La seconda dimensione di cui è portatore il manuale è la trasposizione metodologica. Il manuale può essere considerato la materializzazione del passaggio dal sapere sapiente al sapere insegnato [Chevallard 1991], ma tale trasformazione è filtrata alla luce della normativa scolastica vigente, delle concezioni e dei valori dominanti nella comunità professionale, delle mode metodologiche del momento. Pertanto, accanto alla dimensione del sapere, è presente la dimensione della mediazione del sapere, messo a disposizione già pronto per essere calato nella pratica, attraverso precisi ma non sempre giustificati metodi e strumenti che di conseguenza perdono l'aderenza al contesto di apprendimento e diventano modellizzazioni astratte, consuetudini più che vere e proprie scelte didattiche [Pentucci 2018].

Infine, il manuale porta con sé anche una dimensione pedagogica della trasposizione:

«il ruolo primario della scuola o di un manuale scolastico, per quanto concerne l'aspetto etico, è di contribuire a formare dei cittadini moralmente, eticamente e intellettualmente autonomi»

[Shapiro 2007, 84]

\section{La trasposizione del sapere storico: un esempio}

Come viene raccontata la storia nei libri di testo per la scuola di base? L'organizzazione dei saperi è affidata ad un canone che emerge dalle scelte editoriali e collocata in una dimensione temporale profonda, tematizzata e legata a linee interpretative proprie della storiografia esperta?

L'analisi è condotta su un corpus di 10 sussidiari per la scuola primaria, nella parte dedicata alla storia, tutti editi tra il 2012 e il 2017 , di diverse case editrici tra le più presenti nel mondo delle adozioni scolastiche.

In questo contributo si dà conto di un filone parziale di ricerca, facente parte di un progetto più ampio, con focus sulla geostoria, condotto tra gli anni 2017 e 2018 e dedicato sia ai sussidiari, sia ai manuali di storia e geografia della scuola secondaria di I grado [Pentucci 2018].

Il tema dell'osservazione è stato individuato nella periodizzazione: essa è stata rilevata osservando due aspetti dei libri in questione: da un lato il modo in cui il divenire storico viene suddiviso tra $\mathrm{i}$ volumi ed all'interno dei volumi nelle unità, sezioni o capitoli che li compongono; dall'altro le parti dei manuali dedicate espressamente alla periodizzazione ed alla cronologia, per comprendere la linea interpretativa ad essa sottesa.

\section{La periodizzazione come elemento organizzatore del sapere storico}

«Nella costruzione del racconto storico, lo storico dispone, come elementi di base o come pilastri naturali, il tempo e lo spazio. Nel collocare le informazioni fattuali concernenti il passato nel tempo e nello spazio, lo storico, attraverso la concettualizzazione, crea delle totalità narrative» 
Nello specifico il tempo inteso come oscillazione tra passato e presente è l'elemento che conferisce ad un fatto narrato la dimensione della proposizione storica. Il tempo storico è un tempo tanto lineare quanto profondo: $\mathrm{i}$ concetti di successione, durata, periodo devono essere interconnessi a quelli di simultaneità, permanenza e mutamento, contemporaneità, così come va adeguatamente definito il mai netto confine tra fatto e processo e chiarita la ricorsività presente nelle nozioni di ciclo, congiuntura, tendenza.

Il tempo, dunque, è il grande organizzatore del sapere storico ed in tal senso si esplicita nelle tre operazioni di base che permettono di mettere in discorso il sapere nella dimensione temporale del racconto: la datazione, la cronologia e la periodizzazione.

Se la datazione rappresenta la collocazione oggettiva degli eventi lungo un asse temporale, la cronologia è invece una scelta, che investe i concetti di scala, tematizzazione, lungo e breve periodo. La periodizzazione, infine, è un'operazione di carattere interpretativo e cognitivo, che restituisce in maniera sintetica la visione complessiva che lo storico vuole restituire e determina lo sfondo conoscitivo necessario per la ricostruzione storica. La periodizzazione, dunque, ha un portato culturale e filosofico, è fortemente soggettiva, o comunque prospettica, ed assolve, rispetto alla conoscenza storica, a scopi differenti: da un lato ha una finalità didattica che serve a scandire la storia nei programmi e, di conseguenza, nei manuali ed ha il suo prodromo e consolidamento nella tradizione scolastica; risolve, inoltre, il problema storiografico di correlare fenomeni culturali complessi con le rispettive connotazioni cronologiche che ne sono anche denotative (es.: il Rinascimento). Infine, risponde ad una esigenza di immediatezza comunicativa che renda riconoscibile un'età correlata ad un personaggio o evento (es. l'età di Pericle) o ad una generazione (es.: il Sessantotto).

Poste queste premesse, è evidente che la periodizzazione presenta dei limiti, in quanto schematica, convenzionale ed arbitraria; tuttavia è indispensabile per dare ordine al passato e renderlo intellegibile.

Poiché si tratta di una forma di interpretazione, avendo carattere di azione umana sul tempo essa è mobile e situata, ovvero può essere messa in discussione, rivista e superata.

Sul piano didattico e scolastico è imprescindibile per dare ordine ai saperi storici e, nello stesso tempo, per costruire una concettualizzazione dell'idea di tempo, che non emana direttamente dall'insegnamento della storia ma richiede interventi didattici mirati. Altrettanto importante è presentare una pluralità di possibili cronologie per evidenziarne il carattere problematizzante, che rende intellegibile e decifrabile il mutamento e permette di interpretare o comunque di riconoscere le interpretazioni ad essa sottese, poiché esprimendo l'idea di un passaggio rappresenta il disconoscimento nei confronti delle idee, dei valori, della società del periodo precedente [Le Goff 2014].

Per la sua funzione di organizzazione del sapere la periodizzazione è un elemento fondante nella struttura dei manuali di storia, che ne rispecchiano, anche nella suddivisione del divenire storico in volumi e nei relativi anni scolastici cui sono destinati, la scelta e la segmentazione dell'asse temporale di riferimento.

Le Indicazioni Nazionali forniscono una periodizzazione di massima, suggerendo un'articolazione che nella scuola primaria comprende le conoscenze situabili tra la comparsa dell'uomo e la tarda antichità, mentre nella scuola secondaria di I grado deve essere affrontato il periodo tra la tarda antichità e gli inizi del XXI secolo, con la specificazione di dover dedicare l'ultimo anno allo studio del Novecento. 
È comunque successivamente specificato che non è necessario «soffermarsi troppo a lungo su singoli temi e civiltà remote, nella convinzione che in una data classe si debbano svolgere solo argomenti specifici» [Indicazioni Nazionali, 53], ma si invita fortemente ad utilizzare un approccio che prenda sempre in considerazione il presente e le problematiche contemporanee legate ai concetti geostorici di base, a prescindere dall'annualità di riferimento.

L'approccio cronologico, dunque, pur suggerendo una generale ripartizione della storia, è piuttosto elastico e la curricolazione del sapere storico nel divenire temporale potrebbe essere varia ed orchestrata secondo linee storiografiche differenti, legata a canoni di riferimento recenti ed improntati ad una visione di world history e basata su un percorso didattico presente - passato ritorno al presente in grado di attualizzare i fenomeni storici e renderli funzionali alla comprensione del mondo ed alla strutturazione del pensiero storico propriamente detto [Bloch 2015].

\section{Una mancata periodizzazione nei libri per la scuola primaria}

I manuali in realtà presentano una linearizzazione del sapere che prende in carico una dimensione di tempo quasi esclusivamente vettoriale, diversamente intesa nella scuola primaria e nella scuola secondaria di I grado.

Nella scuola primaria, infatti, il discorso della periodizzazione rimane implicito, non dichiarato nella divisione dei volumi, ma in generale organizzato in una trattazione molto generica della storia della terra senza l'uomo e della comparsa dell'uomo sulla terra fino ai primi esempi di domesticazione in classe terza, della neolitizzazione osservata nel periodo tra il 3500 a.C. e la nascita di Cristo, ma reificata attraverso una successione di civiltà non periodizzate in classe quarta, per poi tornare al primo millennio avanti Cristo e descrivere altre civiltà in un ordine non determinato da elementi di tipo temporale, né esaustivo sul piano spaziale, per fermarsi alla fine del IV sec. d.C. prendendo in carico la divisione dell'Impero Romano tra Oriente ed Occidente.

Questa organizzazione dei saperi non è corretta sia a livello di tempo che di spazio: infatti, non si può parlare di una vera periodizzazione in quanto si giustappongono descrizioni di popolazioni collocate nel tempo secondo procedure che non rispettano i principi della datazione sopra esposti, ma utilizzano espressioni rimandanti ad un tempo vago e non precisamente definito, come nell'esempio seguente: 


\section{ASCESA E DECLINO DEGLI ETRUSCHI}

All'inizio della loro storia gli Etruschi occupavano solamente il territorio che corrisponde alla Toscana, alla parte occidentale dell'Umbria e a quella settentrionale del Lazio.

Poi, verso il VII e il VI secolo a.C., estesero il loro dominio anche ad altre regioni italiane.

A nord, attraversarono l'Appennino, occuparono una parte della Pianura padana e si spinsero fino alle coste del Mar Adriatico, fondando molti nuovi insediamenti, tra cui Adria, la città che avrebbe dato il nome al mare stesso.

A sud, prima imposero la loro egemonia su diverse città del Lazio (la stessa Roma fu governata da re etruschi per molti anni), poi si spinsero fino in Campania, dove costruirono nuove e fiorenti città.

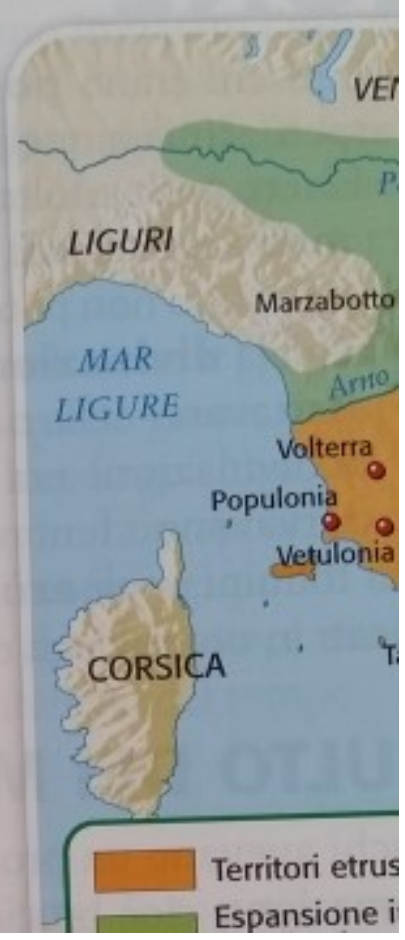

Altra notazione significativa è quella relativa all'avvio della cronologia storica: nonostante le Indicazioni diano un preciso suggerimento consigliando di avviare la sistematizzazione cronologica delle conoscenze dalla comparsa dell'uomo sulla terra, di fatto eliminando il lunghissimo periodo della storia della terra senza l'uomo che invece è presente negli approcci curricolari ispirati alla Big History [Spier 2015], tutti i libri di classe terza primaria fanno partire lo studio della storia dal Big Bang, attraverso una serie di rappresentazioni piuttosto elusive in termini spazio-temporali, che passano da questioni astronomiche a questioni geologiche, a questioni biologiche, fino a raccontare la comparsa dell'uomo, collocato genericamente in universi astratti, attraverso quadri illustrati da scenografie immaginarie, vicine più al «c'era una volta» delle fiabe che ad una effettiva collocazione dei fatti nella storia, impossibile poiché il sistema di datazione è pressoché assente.

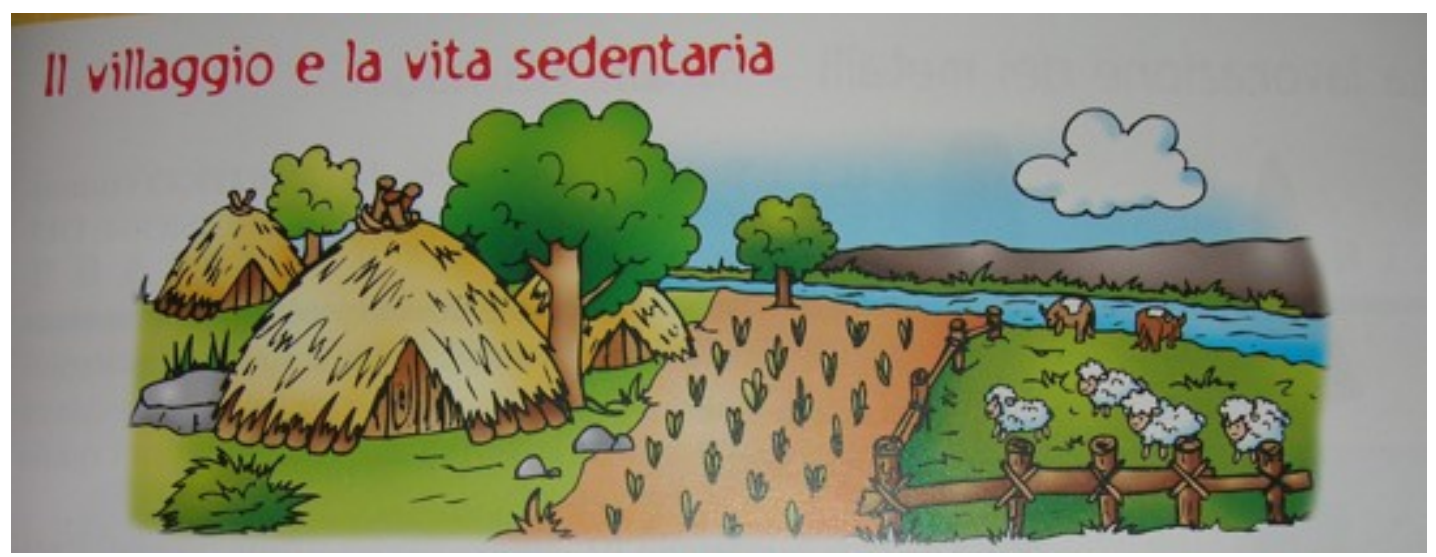


Il problema dell'avvio della storia non è di secondaria importanza. Da un lato è rappresentativo del canone che si intende seguire, in quanto, come già precisato, operare una scelta tra un inizio che preveda la storia dell'universo e della terra senza l'uomo ed uno che si limiti alla storia dell'uomo sulla terra significa allinearsi a prospettive differenti, la prima inquadrata negli studi di Big History e Big Geography, di portata transdisciplinare, che allude ad un portato di storia globale e non solo mondiale e ad una periodizzazione strutturata sul lunghissimo periodo; la seconda invece centra l'attenzione sugli uomini come agenti di storia e sulle relazioni tra uomo e ambiente.

Si può parlare di una sorta di canone interno, sviluppato e consolidato nella comunità scolastica, che trova la sua reificazione nei manuali ma che è fortemente antistoriografico, basato sulla stratificazione di concezioni e luoghi comuni e sul rimaneggiamento di teorie spesso non più valide perché superate dalle scoperte recenti. Quali sono le fonti di tale canone? Da un lato esso deriva da una concezione nazionalistica, propria degli stati che devono consolidare, anche attraverso il discorso storico, la propria identità e quella dei propri cittadini. L'identificazione fra storia e civiltà infatti consente di «presentare la nazione come il decantato delle civilizzazioni passate» [Brusa $2007,65]$, contrapponendo una preistoria ferina e incivile ad una storia che invece rappresenta una progressiva conquista della civiltà.

La base normativa che scandisce tale canone va cercata in un passato più recente: la scelta degli argomenti e la loro successione tra i vari anni di scuola primaria è quella dei programmi Moratti, del 2004, ultimo documento ministeriale che abbia declinato anno per anno i contenuti obbligatori da affrontare nelle varie discipline.

Disatteso è invece l'invito delle indicazioni del 2012 ad occuparsi anche del presente della storia. Solo alcuni sussidiari inseriscono schede o sezioni, avulse dalla narrazione storica vera e propria, attraverso le quali trattano alcune questioni generiche come il concetto di democrazia oggi, le olimpiadi moderne, la descrizione attuale di alcuni territori o stati. Il criterio è sempre quello analogico, non si tratta di un percorso che parta dalla problematizzazione di elementi della contemporaneità affrontati attraverso una ricostruzione storica che ne spieghi le cause e le configurazioni, ma di una attualizzazione di superficie, in cui lo stesso presente non è un tempo storico, ma una dimensione generica e non contestualizzata.

\section{Conclusioni}

La doppia valenza del manuale di strumento mediatore per la strutturazione dei saperi negli studenti e di strumento organizzatore per la progettazione dell'insegnante è sicuramente la caratteristica più evidente.

Inoltre, la sua già affermata centralità nella realtà delle prassi scolastiche e l'identificazione di esso con il sapere scolastico rende quantomai necessario prenderlo in considerazione come elemento assolutamente integrato del processo di insegnamento - apprendimento e perciò farsene carico nel momento in cui si porta l'attenzione al polo dell'oggetto culturale, la cui dimensione è fortemente implicata con la tipologia di sapere e le modalità di mediazione proposte dal manuale.

\section{Bibliografia}


- Bloch M., 2016, Apologia della storia o Mestiere di storico, a cura di Panizza C., Alessandria: Falsopiano (ed. or. 1949)

- Brusa A. 2007, Gli stereotipi colti sulla preistoria, in Sarti L. e Tarantini M. (acd.), Evoluzione, preistoria dell'uomo e società contemporanea (pp. 56-67), Roma: Carocci

- Chervel A. 1998, La culture scolaire. Une approche historique, Paris: Belin

- Chevallard Y. 1991, La transposition didactique, du savoir savant au savoir enseigné. Edition augmentée, Grenoble: La Pensée Sauvage

- Le Goff, J. 2014, Il tempo continuo della storia, Roma-Bari: Laterza

- Lenoir Y. (2006), Du curriculum formel au curriculum enseigné: comment des enseignants québécois du primaire comprennent et mettent en ouvre le nouveau curriculum de l'enseignement primaire, in Audigier, F., Crahay M. e Dolz J. (acd), Curriculum, enseignement et pilotage (p. 119-141), Bruxelles: De Boeck Université

- Norman D. 1993, La caffettiera del masochista. Psicopatologia degli oggetti quotidiani, Firenze: Giunti

- Pentucci M. 2018, Come da manuale. La trasposizione didattica nei contesti d'insegnamento-apprendimento, Reggio Emilia: Junior

- Shapiro L. 2007, The embodied cognition research programme, "Philosophy compass", 2(2), 338-346

- Spier F. 2015, Big history and the future of humanity, Hoboken: John Wiley \& Sons

- Topolski J. 1997, Narrare la storia. Nuovi principi di metodologia storica, Torino: Pearson Italia

- Vargas L. 2006, Transnational Media Literacy: analytic reflections on a program with Latina teens, "Hispanic Journal of Behavioral Sciences", 28(2), 267-285 